\title{
Situated Interaction in Ubiquitous Computing
}

\author{
Albrecht Schmidt \\ Telecooperation Office ( $\mathrm{TecO}$ ) \\ University of Karlsruhe \\ Vincenz-Priessnitz-Str. 1 \\ 76131 Karlsruhe, Germany \\ +49721690229 \\ albrecht@teco.edu
}

\author{
Walter Van de Velde \\ Starlab Nv/Sa, \\ Excelsiorlaan 40-42 \\ 1930 Zaventem, Belgium \\ +3227215454 \\ wvdv@starlab.net
}

\author{
Gerd Kortuem \\ Computer Science Department \\ University of Oregon \\ Eugene, OR 97403, USA \\ $+15413461381$ \\ kortuem@cs.uoregon.edu
}

\section{Keywords}

Context awareness, information appliances, situated interaction, ubiquitous computing, wearable computing.

\section{INTRODUCTION}

The situations in which human-computer interaction takes place are increasingly varied, as computers become highly portable and embedded in everyday environments. Research reported from different communities (Wearable Computing, Mobile Computing, HCI, CSCW, Augmented Reality) indicates that awareness of situations can lead to improvement of human-computer interaction. We propose a workshop at CHI2000 to provide a forum to discuss situational awareness and situated interaction.

With the availability of sensing technologies, such as measuring the surrounding light conditions, the motion of the user, the orientation of a display, users' position relative to an information appliance, the number of users in front of a device, users' emotional state (bio-sensors), etc., this situational context can be captured and used as additional input to the system. The interaction process can benefit from the additional knowledge about the situation [1].

\section{THEMES AND TOPICS}

In the design and development of interactive devices that are used in various environments and different situations the classical interaction paradigms as know from desktop applications are often not appropriate. The situation and the surrounding environment provide information that should be taken into account by the applications as additional input. In the workshop we explore the impact of situational awareness on human computer interaction. We will focus especially on the following topics:

- Automated extraction and identification of the situational context from sensory data.

- Adaptation of input and output modalities according to usage situation and recognized requirements [2,3].

- Reduction in the need for explicit user input through automated information capture and reasoning.

- Determination the right time and mode for interrupting the user appropriate to the situation [4].

Situational awareness is especially attractive for two classes of devices, see Fig 1. First ultra-mobile devices, characterized by the fact that these devices are operated and operational while on the move, most notably: wearable computers, smart mobile phone, handheld computers and
PDAs. Second for shared stationary devices in common spaces or in changing environments e.g. public displays, meeting rooms, shared offices.

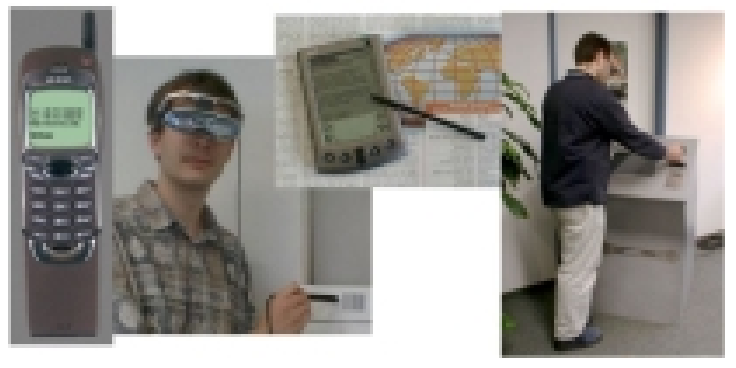

Fig.1: Mobile and stationary devices.

With currently available technology different mechanism are given to detect situational context, namely: sensors for physical, chemical, and biological parameters, tools for capture and analysis of video and audio data.

\section{GOALS}

The main goal of the workshop is to develop an understanding of how the situation of use does influence the interaction process. We will provide a forum to share information, results, and ideas on current research in the area of context awareness and situated computing with its respect to human computer interaction. Furthermore we aim to develop new ideas on how to exploit context for improving human computer interaction and to identify possible obstacles on that way. Results will be available at http://www.teco.edu/chi2000ws/.

\section{References}

1. Abowd, G.D., Atkeson, C.G, Brotherton, J.A., Enqvist, T., Gully, P., Lemon, J. Investigating the capture, integration and access problem of ubiquitous computing in an educational setting. In Proceedings of CHI '98, May, (1998).

2. Kortuem, G., Segall, Z., Bauer, M. Context-Aware Adaptive Wearable Computers as Remote Interface to Intelligent Environments. Proceedings of the 2nd International Symposium on Wearable Computers. Pittsburgh. 1998.

3. Schmidt, A., Aidoo, K. A., Takaluoma, A., Tuomela, U., Van Laerhoven, K., Van de Velde, W.. Advanced Interaction in Context. 1st International Symposium on Handheld and Ubiquitous Computing (HUC99), Germany, Sept. 1999.

4. Sawhney, N., Schmandt, C. Nomadic Radio: Scaleable and Contextual Notification for Wearable Audio Messaging. In Proceedings of the 1999 Conference on Human Factors in Computing Systems (CHI '99), Pittsburgh, PA, May 15-20, 1999. 